\title{
Ambient Optomechanical Alignment and Pupil Metrology for the Flight Instruments aboard the James Webb Space Telescope
}

\author{
Phillip Coulter ${ }^{1}$, Alexander Beaton ${ }^{3}$, Jeffrey S. Gum ${ }^{1}$, Theodore J. Hadjimichael ${ }^{1}$, Joseph E. \\ Hayden $^{8}$, Susann Hummel ${ }^{4}$, Jason E. Hylan ${ }^{1}$, David Lee ${ }^{10}$, Timothy J. Madison ${ }^{1}$, Michael \\ Maszkiewicz ${ }^{2}$, Kyle F. Mclean ${ }^{7}$, Joseph McMann ${ }^{7}$, Markus Melf ${ }^{4}$, Linda Miner ${ }^{1}$, Raymond G. OhI ${ }^{1}$, \\ Kevin Redman ${ }^{7}$, Andreas Roedel ${ }^{4}$, Paul Schweiger ${ }^{6}$, Maurice Te Plate ${ }^{5}$, Martyn Wells ${ }^{10}$, Greg W. \\ Wenzel $^{7}$, Patrick K. Williams ${ }^{9}$, Jerrod Young ${ }^{1}$ \\ ${ }^{1}$ NASA Goddard Space Flight Center \\ ${ }^{2}$ Canadian Space Agency \\ ${ }^{3}$ Comdev International \\ ${ }^{4}$ EADS Astrium Gmbh \\ ${ }^{5}$ European Space Agency \\ ${ }^{6}$ Lockheed Martin Corporation \\ ${ }^{7}$ Qinetiq North America \\ ${ }^{8}$ Sigma Space Corporation \\ ${ }^{9}$ SGT Inc \\ ${ }^{10}$ UK Astronomy Technology Centre
}

\begin{abstract}
The James Webb Space Telescope science instruments are in the final stages of being integrated into the Integrated Science Instrument Module (ISIM) element. Each instrument is tied into a common coordinate system through mechanical references that are used for optical alignment and metrology within ISIM after element-level assembly. In addition, a set of ground support equipment (GSE) consisting of large, precisely calibrated, ambient, and cryogenic structures are used as alignment references and gauges during various phases of integration and test (I\&T). This GSE, the flight instruments, and ISIM structure feature different types of complimentary metrology targeting. These GSE targets are used to establish and track six degrees of freedom instrument alignment during I\&T in the vehicle coordinate system (VCS). This paper describes the optomechanical metrology conducted during science instrument integration and alignment in the Spacecraft Systems Development and Integration Facility (SSDIF) cleanroom at NASA Goddard Space Flight Center (GSFC). The measurement of each instrument's ambient entrance pupil location in the telescope coordinate system is discussed. The construction of the database of target locations and the development of metrology uncertainties is also discussed.
\end{abstract}

Keywords: JWST, ISIM, NIRSpec, NIRCam, FGS, MIRI, metrology 


\section{INTRODUCTION}

The James Webb Telescope (JWST) is a large (6.6m diameter) deployable, segmented telescope that will be used for space-based IR astronomy ${ }^{1}$. The JWST science payload consists of the Optical Telescope Element (OTE) and the Integrated Science Instrument Module (ISIM) ${ }^{2}$. The ISIM element contains four science instruments (SI). The ISIM structure is comprised of a low CTE composite material. The JWST mission involves partners from multiple countries and organizations. ESA is providing the launch vehicle, the Near Infrared Spectrometer (NIRSpec) ${ }^{3}$. The Canadian Space Agency (CSA) is providing the Fine Guidance Sensor (FGS) ${ }^{4}$. Lockheed Martin and the University of Arizona are providing the Near-Infrared Camera (NIRCam) ${ }^{5}$. The Mid-Infrared Instrument was built by a European Consortium, led by the UK Astronomy Centre, together with JPL who are responsible for flight software, detectors and cooler ${ }^{6}$. NASA is providing the ISIM and OTE elements.

The science instruments are in the process of being integrated to the ISIM structure and tested at the Goddard Space Flight Center (GSFC) Spacecraft Systems Development and Integration Facility (SSDIF). Each of the instruments are aligned to a common vehicle coordinate system (VCS) via various mechanical references on the ISIM structure and the instruments themselves. In addition to this there are several ground support equipment (GSE) components consisting of large, precision-calibrated, ambient, and cryogenic structures used as alignment references and gauges during various phases of integration and test (I\&T). These GSE targets are used to establish and track instrument alignment during I\&T. At the time of this publication the ambient testing of ISIM with all science instruments integrated into the ISIM structure, prior to the second iteration of cryogenic vacuum testing (CV2), has been completed. The first iteration of cryo-vac testing (CV1) testing, with only MIRI and FGS installed, was completed in the fall of 2013.

\section{PURPOSE OF WORK}

The purpose of this work is to verify the ambient alignment of the science instruments (SI) to the integrated science instrument module (ISIM) structure. This work checks that the SIs are aligned mechanically to the Vcoordinate system (VCS) within the system requirements. At the cryogenic operating temperature of JWST, instrument alignment is different and is verified via optical testing using a telescope simulator. The VCS is the global telescope coordinate system. The VCS origin is located at a virtual point on the nominal, on-orbit, cryogenic primary mirror vertex. The orientation of the VCS coordinate system is shown in Figure 1. 


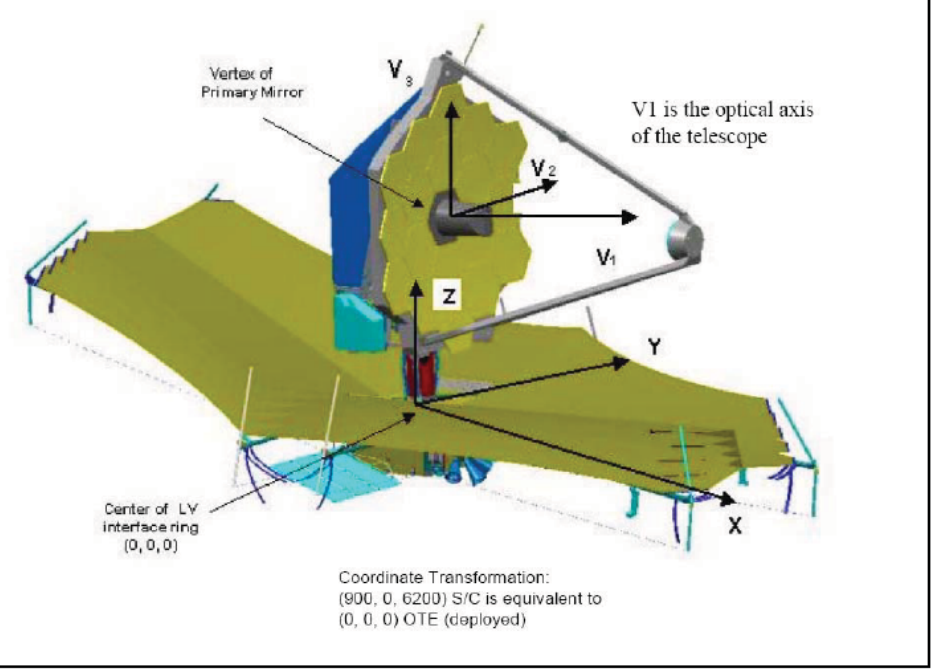

Figure 1 JWST V-coordinate system definition.

The ISIM structure and SIs are equipped with various ground support equipment (GSE) laser tracker (LT) and laser radar (LR) metrology nests as well as alignment cubes. The nests provide a six degree of freedom reference. The cubes provide a check on angular alignment. During this work, the ISIM structure is attached to the ISIM Test Platform (ITP). The ITP simulates the Optical Telescope Element (OTE) mechanical interface which features LT/LR nests as well as several alignment cubes. The SI nests are located at each instruments optical bench (OB) tooling holes. In addition to the mechanical targets, each SI contains a Pupil Alignment Reference ${ }^{7}$ (PAR) that is located at the internal pupil stop of each instrument. The image of each PAR is measured near the ambient entrance pupil location. Each SI prior to integration and during their build up was attached to a calibrated structure that simulates the SI-ISIM interface, the Ambient Science Instrument Mechanical Interface Fixture (ASMIF). The ASMIF places the instruments with V1 approximately horizontal (i.e., perpendicular to gravity) and V2 approximately parallel with gravity. Custom shims called Science Instrument Interface Plates (SIIP) were fabricated to place the SIs at their nominal ambient location in VCS. Matching SIIP plates were fabricated for the ASMIF and ISIM structure.

Alignment verification is done by checking the 6-DOF mechanical alignment of each SI OB with respect to 8 reference targets on a stable portion (on the +V1 side of ISIM) of the ISIM structure called the "B-reference". There is also an optomechanical reference called the "A-reference". These A-reference targets are located at the OTE-ISIM kinematic feet interface on the OTE side. For the work presented the B-reference targets are appropriate to use. The B-reference targets are the most stable targets on ISIM at ambient and cryogenic temperatures and for all gravity orientations and load conditions. This was done by using a network of LT/LR and theodolites. The PAR for each science instrument was measured with an alignment telescope technique. A finite element model (FEM) ${ }^{8,9}$ is used to predict the sag of ISIM associated with making measurements in a 1-g state to effectively remove the g-release effect from the metrology results predicting an as-built, ISIM model for a 0-g, onorbit state. 


\section{REQUIREMENTS}

The requirements derived are a bottoms-up uncertainty estimation that inform a system wide error analysis where comparison to the top-level ISIM requirements is made. The overall test requirement for the metrology is that the measured SI OB locations (nests and cubes) are at their predicted locations to within the 2-sigma uncertainties of:

1. The SI OB measured locations with the SIs integrated to the ISIM structure.

2. The SI OB measured locations while integrated to the ASMIF structure with FEM and SIIP deltas applied.

In theory the SI-ASMIF interface is supposed to be identical to the ISIM-SI interface. In reality, there are slight differences in the SI-ASMIF and ISIM-SI interfaces. This feeds into the pass/fail requirements for SI locations on the ISIM structure. Two factors must be considered. First, the uncertainty in the as-built alignment of the $\mathrm{SI}$ in the ambient, loaded ISIM structure under the 1-g condition. The second factor is the as-built SI OB location uncertainty. The values should agree within the uncertainties of these two factors. A grass roots uncertainty estimate is given in Figures 2 and 3. All values in the diagram are two-sigma. Each SI has slightly different pass/fail values. Each factor in the block diagram is described as follows. The "SI on ASMIF" is the measurement uncertainty of the OB measurements with the SI integrated to the ASMIF. The "Transformation" is the uncertainty in fitting to the as-built ASMIF values. The "ASMIF TB" is the measurement uncertainty of the ASMIF nests which define the VCS. The "Total ASMIF" is simply the RSS of the three factors just discussed. The "Total SIIP" is the RSS of the ISIM and ASMIF SIIP calibration uncertainties. The "SI on ISIM" is the measurement uncertainty of the ISIM targets. The "Transformation" (on the left side of the diagram) is the uncertainty in fitting the measured values to the as-built values. The "B-ref" is the measurement uncertainty of the ISIM B-reference targets. The "Total ISIM" is the RSS of the three factors just discussed. The total SI pass/fail value is the RSS of the four factors below in the block diagram. The RSS is used because the uncertainty distributions are assumed to be random and uncorrelated.

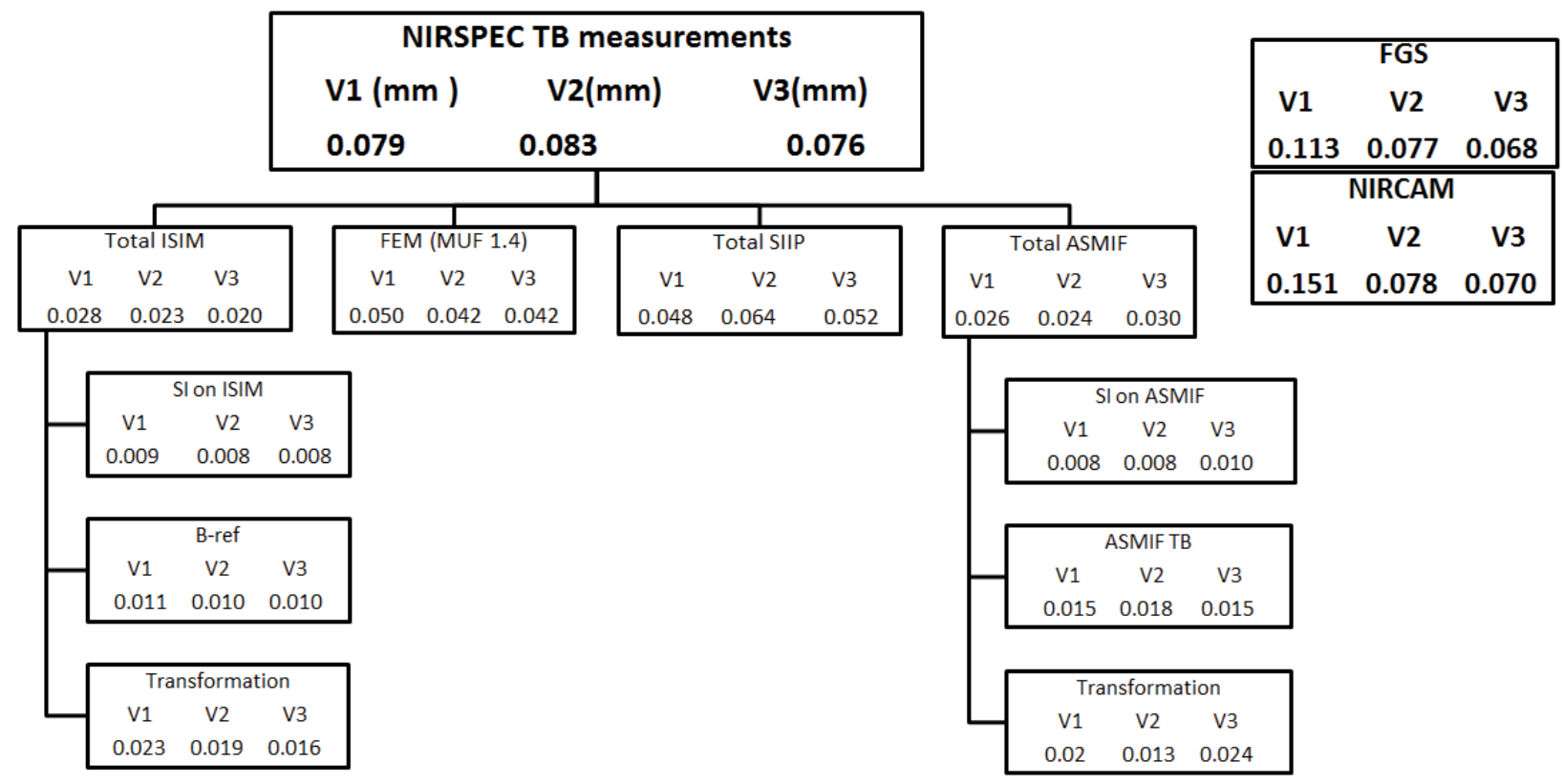

Figure 2 Block diagram showing the factors involved in determining the pass/fail values for some representative SIs. 


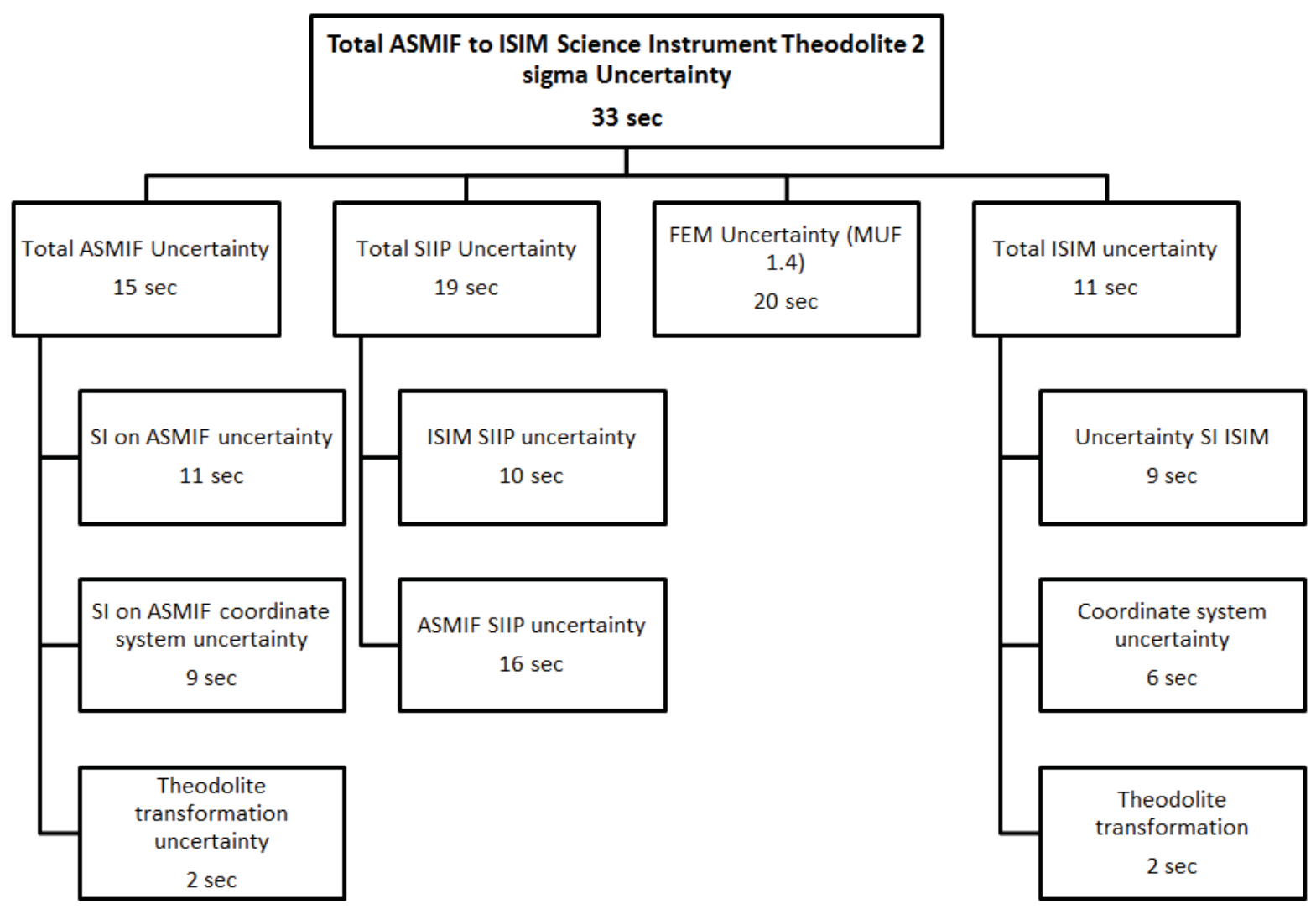

Figure 3 Uncertainty block diagram for theodolite measurements applicable to all SIs.

The PAR requirements are based on relative test-to-test changes. The PAR image position change can indicate possible internal movement of elements within each SI between pre- and post-environmental testing and is also sensitive to bulk SI rotation. The requirement of the test-to-test PAR position change is simply the RSS of the individual test uncertainties. The individual test uncertainties were estimated from several factors. Such factors include errors in some of the GSE used in the test setup, LT measurements of the spherically mounted retroreflector (SMR) defining the pupil point and uncertainties in the image analysis. The pass/fail values are listed in Table 1 below.

Table 1 Test to test pass/fail values for the PAR image location.

\begin{tabular}{|l|r|r|r|}
\hline \multirow{2}{*}{ Test to test uncertainty $(2 \sigma)$} & V1 $(\mathrm{mm})$ & $\mathrm{V} 2(\mathrm{~mm})$ & $\mathrm{V} 3(\mathrm{~mm})$ \\
\cline { 2 - 4 } & 0.071 & 0.239 & 0.239 \\
\hline
\end{tabular}

To put this pupil shear trending into perspective, the ISIM level requirement ${ }^{10}$ states that the pupil shear caused by ISIM misalignment and uncertainties shall not exceed 3.1\%. In terms of percent pupil shear, the uncertainties in Table 1 are $0.16 \%$ in V2 and V3. This is found by 


$$
\% \text { Pupil Shear }=100 \frac{\Delta V}{152} \mathrm{~mm}
$$

where $\Delta \mathrm{V}$ are the V2/V3 values in Table 1 and $152 \mathrm{~mm}$ is the approximate nominal exit pupil diameter of the telescope. We make the assumption that a relative movement of the PAR target is the same as a change in pupil shear. The pass/fail criteria in Table 1 is almost a factor of twenty better than the overall requirement.

\section{METHODS AND TOOLS}

A variety of measurement tools are used throughout the measurement suite depending on the requirements and application. The tools used include Leica 840, 500 and 401 laser trackers (LT) ${ }^{11,12}$, a Nikon MV224 laser radar $(\mathrm{LR})^{13,14}$, Leica Wild T3000 autocollimating theodolites, and a Koll Morgen K-122 alignment telescope. The LT is a point like coordinate measurement instrument which utilizes an interferometric (IFM) relative distance or absolute distance meter (ADM) devices, along with angular azimuth and elevation encoders. The angular encoders and the range results allow the measurement of a SMR. These SMRs attach with high repeatability to the nests on the object being measured. The measurement uncertainty is approximately 10-25 microns (1-sigma) per meter of range along the horizontal and vertical (orthogonal to range). The laser radar uses LIDAR principles to measure range and contains azimuth and elevation encoders similar to the LT. The LR measures the center points of specular tooling balls (TB). These tooling balls can be interchanged with the SMRs used by the laser trackers. The $L R$ is also capable of scanning diffuse and some specular surfaces. The range uncertainty of the LR is approximately 15 microns (1-sigma) per meter of range. The uncertainty in the horizontal and vertical directions is approximately the same as the LT. The LR is typically used to measure the ISIM structure due to its no-contact method. The use of an LR rather than an LT minimizes human interaction with the structure because 1.) The LR uses tooling balls that are inexpensive compared to SMRs so the entire structure can be populated once and not disturbed throughout the measurements 2.) The tooling balls do not require repointing when moving from station to station. The LR has also been used to measure some types of thermal blanketing for envelope requirements.

Autocollimating theodolites are typically used for ISIM structure and SI cube measurements. Typical 1-sigma uncertainties are on the order of 10 arcseconds.

In general, five sets of measurements are taken for all types of measurements. This provides a good estimate of the as-measured uncertainties and allows enough sets to drop any outliers. The LT and LR are used measurements are made from multiple stations (positions) around the structure at varying heights. This helps to reduce any systematic uncertainties in the measurements.

\section{MEASUREMENT SETUP}

With all of the SIs integrated to the ISIM structure, LR measurements of the ISIM, ITP and SI nest locations were conducted from multiple stations around the structure. During the LR measurements, theodolite measurements were made of the ISIM, ITP and SI cubes nearly simultaneously. Two orthogonal faces from each cube were measured with a network of theodolites. The setup is shown in the Figure 4 below. 


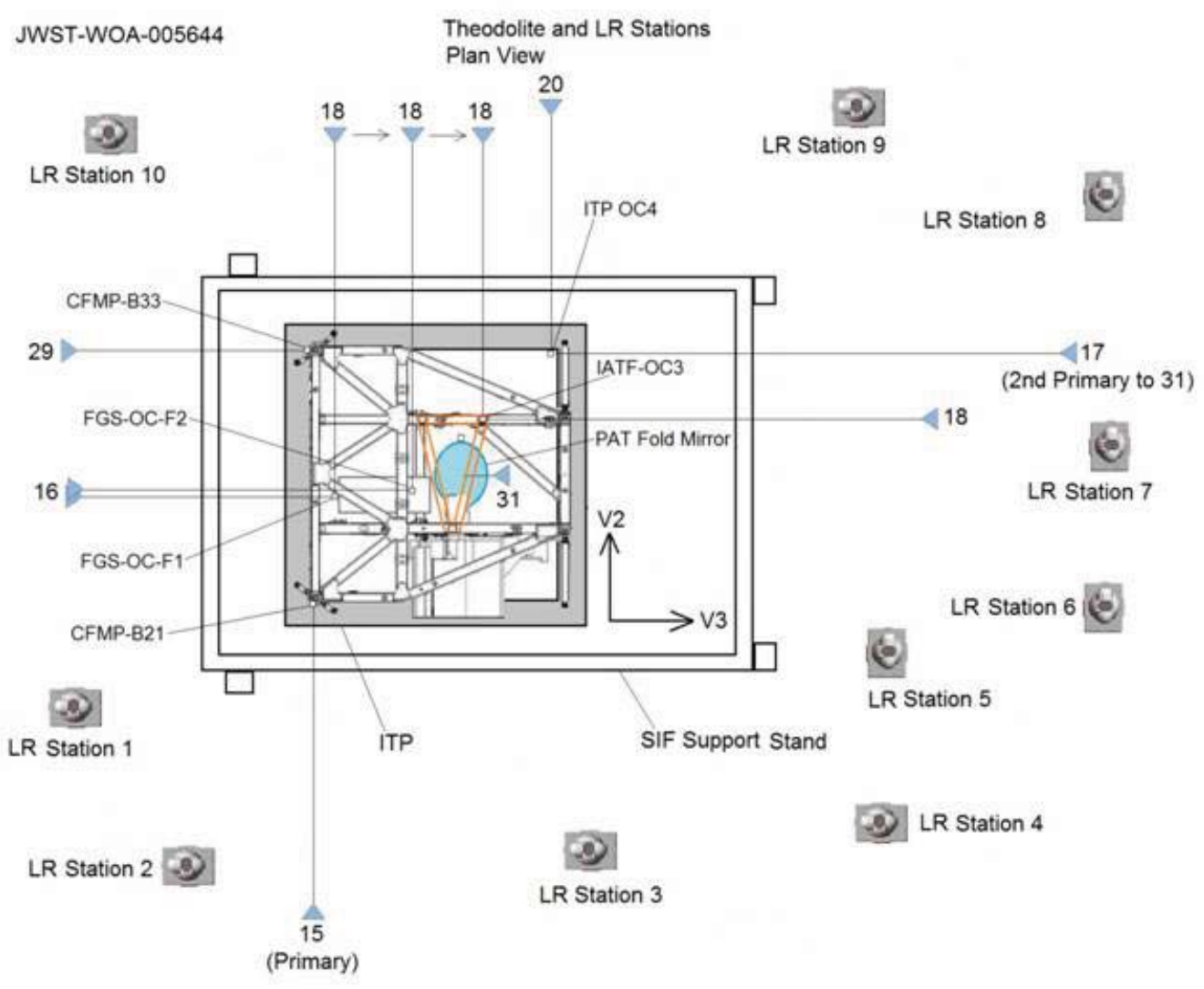

Figure 4 Measurement setup indicating all LR and theodolite stations. The triangles represent theodolite positions.

The PAR measurement is made via an alignment telescope (AT) technique (Figures 5 and 6 ). A fold mirror is used to gain easy access to the chief rays and pupil points of the Sls. A breadboard setup was developed to minimize alignment time and obtain repeatable measurements between tests. The breadboard nest targets are measured and used to realign the bench to VCS values used in previous subsequent tests. The AT is controlled by stages with a digital readout for position. Once the bench is in position the AT can be aligned to each chief ray quickly. The AT is aligned to the nominal ambient chief ray for each instrument. This is done by aligning to two points. The two points are aligned by tracking an SMR (with a LT) in a nest which is mounted to a linear stage with 3-DOF adjustability. The LT is placed in a position that allows it to view the chief ray points as well as other VCS tie-point reference targets on the ISIM and ITP. The first point is positioned at the nominal ambient SI entrance pupil location. The second point is placed somewhere on the chief ray about 1 meter from the pupil point towards the AT. Once the points are adjusted to within about 25 microns to the targeted value the SMRs are replaced with theodolite dot targets. These dot targets contain a small, high-contrast dot placed at the center of the sphere which is co-located with the center or apex of the SMR. The dot targets serve as a visual aid to the AT alignment. In order to simplify the image analysis a third 0.5-inch SMR (i.e., a clocking reference) is positioned next to the pupil point and adjusted along the folded V3 axis until it is aligned with the V2 axis value of the pupil point location. The SMR is replaced with a dot target. The AT is clocked in its dovetail mount until the horizontal crosshair is aligned with the dot. This ensures that the horizontal and vertical crosshairs are in line with the V2 and V3 axis, respectively. 


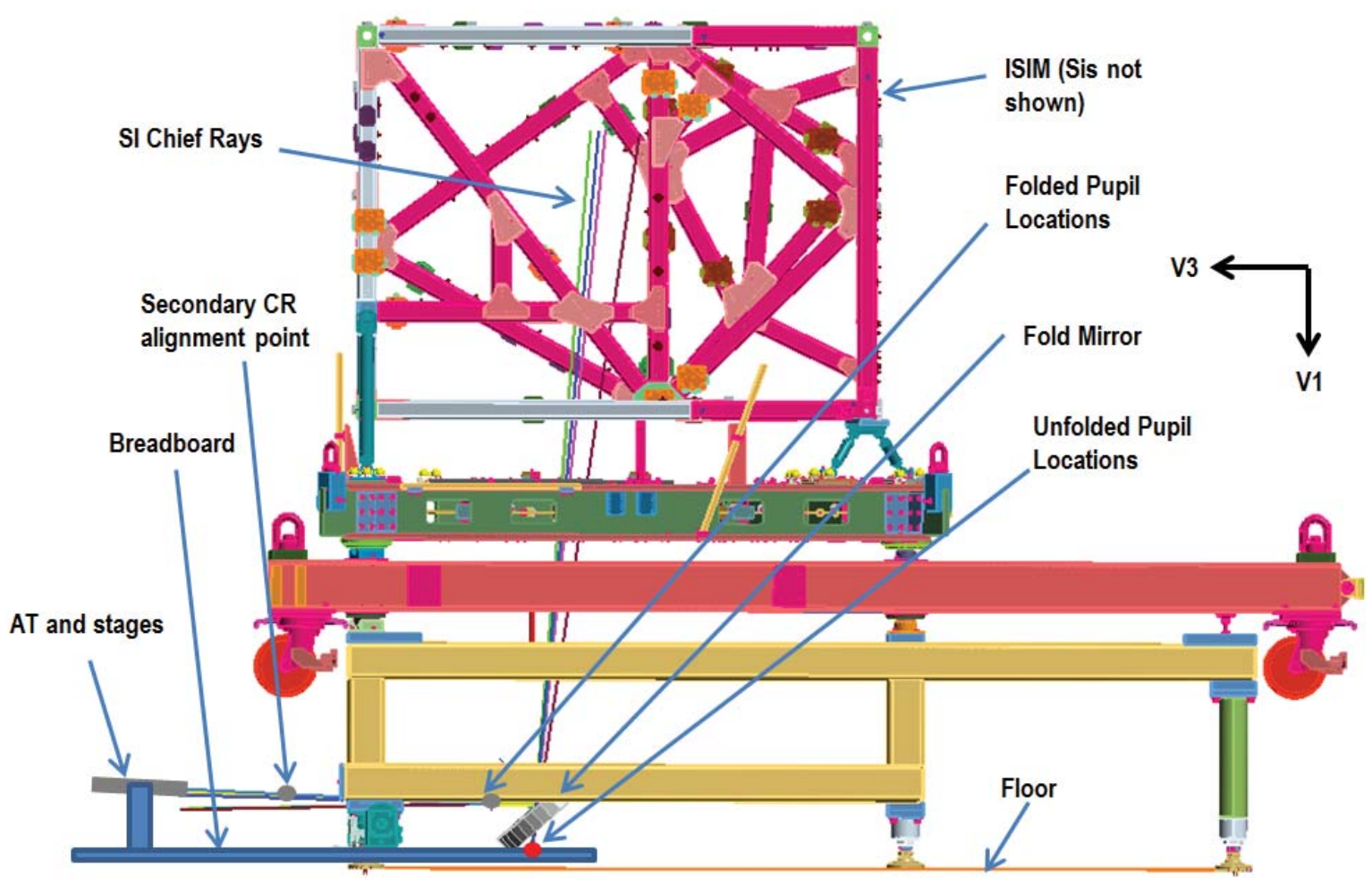

Figure 5 PAR measurement setup. ISIM (SIs not show) is attached to the ITP and test stand. The PAR GSE is located underneath the structure on the floor.

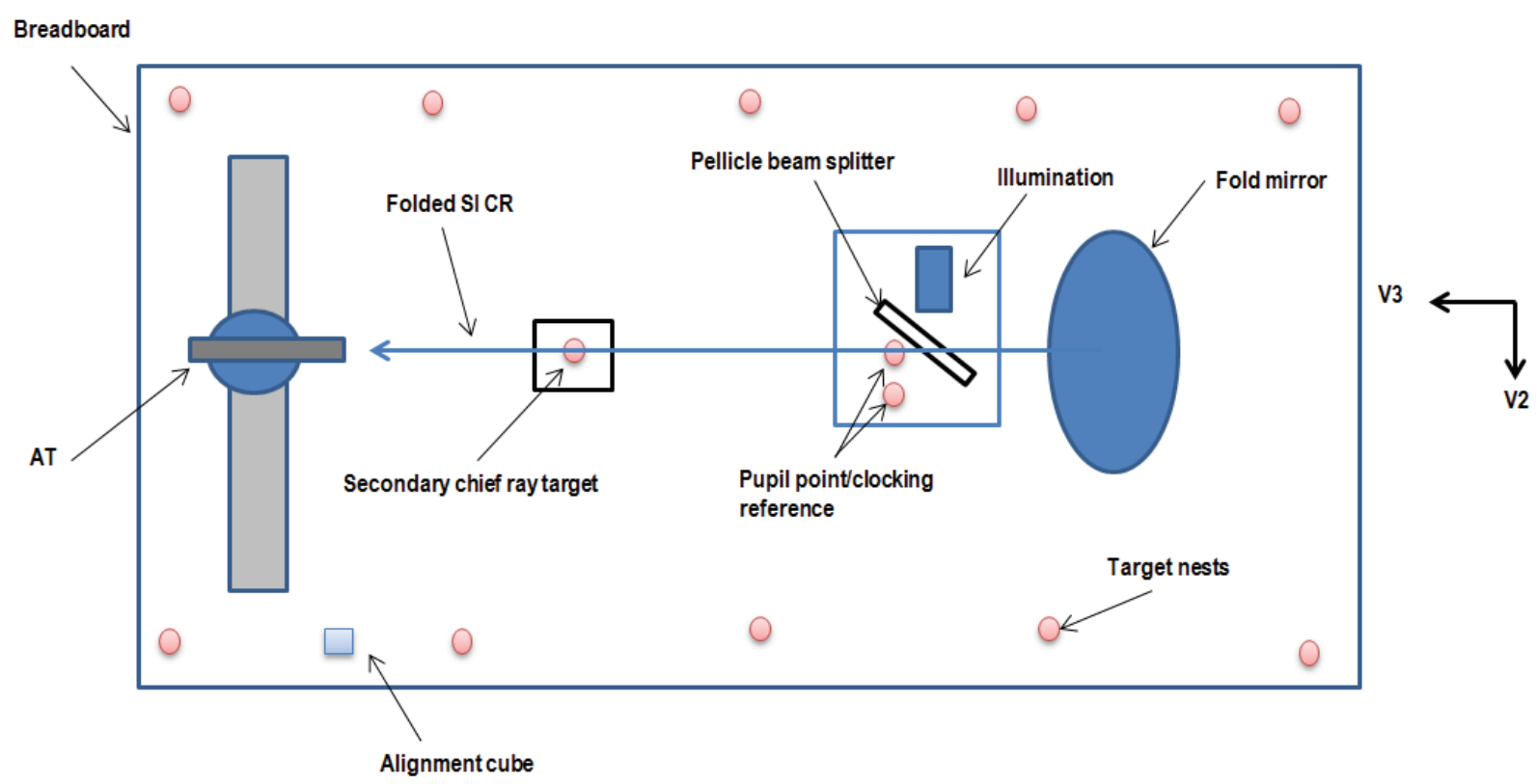

Figure 6 Top view of the PAR measurement GSE breadboard.

Once the alignment is completed, the AT is focused on the nominal ambient pupil target. Both dot targets are removed and illumination is placed approximately in-line via a large beam splitter. The SI PAR targets are reflective 
and need to be front illuminated. A large lamp is used for all SIs with a diffuser placed in front (Figure 6). An IR bandpass filter is used for NIRCam and is placed between the CCD mount and the AT eyepiece. The CCD is used to capture PAR images. A graduated imaging scale is placed at the SI entrance pupil location. An image of the scale is captured by the CCD camera and is used to determine the AT crosshair to PAR center offset in millimeters. A dark image is also taken to improve image contrast via image processing. A total of five images are taken for each SI PAR. The illumination is varied slightly for each image to help average out any pupil wander. Once the images are obtained, two laser trackers measure all visible ISIM, PAR breadboard and ITP targets as well as the chief ray points from different stations. This lowers the measurement uncertainty. This process is repeated for each SI PAR.

\section{ANALYSIS}

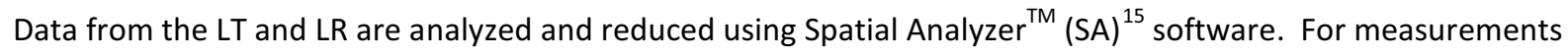
with multiple stations the data are processed and combined using a function in SA called Unified Spatial Metrology Network (USMN) ${ }^{16,17}$. The function is similar to bundling in photogrammetry applications and can reduce the uncertainty of an entire measurement suite. This trilateration-like technique utilizes each instrument's uncertainty in range and angle and combines them in a weighted manner. This function can be used with multiple types of instruments in one network. The theodolite analysis is done using the Optical Alignment Facility Data Analysis Program (OAFDAP) ${ }^{18}$. Surface normal measurements from theodolites are transformed into the VCS using the surface normals established using the direct and through method ${ }^{16}$. The LT/LR determines the surface normal and a point on the mirror by measuring a TB/SMR directly and then through the mirror (i.e., virtual image). Two orthogonal mirrors are leveled to gravity so that their normals are perpendicular to gravity. The same surfaces are measured with theodolites thus linking the measurement systems.

For the TB/SMR measurements of the ISIM, ITP, and SIs, all of the measured nest values from each set are analyzed with the USMN function in SA. Each set is then best-fit transformed to the original calibration of the unloaded ISIM structure. This results in five separate USMN values for each nest location. The students-t $\left(2-\right.$ sigma $^{19}$ and average are calculated in Microsoft Excel using the five data sets. This results in a final averaged location and uncertainty for each measured nest on the ISIM, ITP and SIs.

The PAR analysis for each given SI starts with including each set of data in a USMN of all stations. The chief ray points are included in this process. The final, averaged set of points is best-fit transformed to the final set of ISIM and ITP values from the previous LR measurements of the structure. The resulting, final pupil location represents the center of the alignment telescopes internal crosshair in the image. Image ${ }^{20}$ software is used to calculate the offset from the AT crosshair (with known VCS location) and the PAR target center. Several methods are used to determine the PAR center. The MIRI, NIRSpec, and NIRISS PAR centers are found by simply picking the center point at the vertical and horizontal crosshair intersection. The FGS Guider PAR center is found by fitting an ellipse to the inner circle. The various PAR images are shown in Figure 7. The optimal technique for NIRCam is in progress at the time of this writing. The scale image is used to convert pixels to millimeters. This gives an absolute location of the PAR image in VCS at ambient for trending during I\&T. 

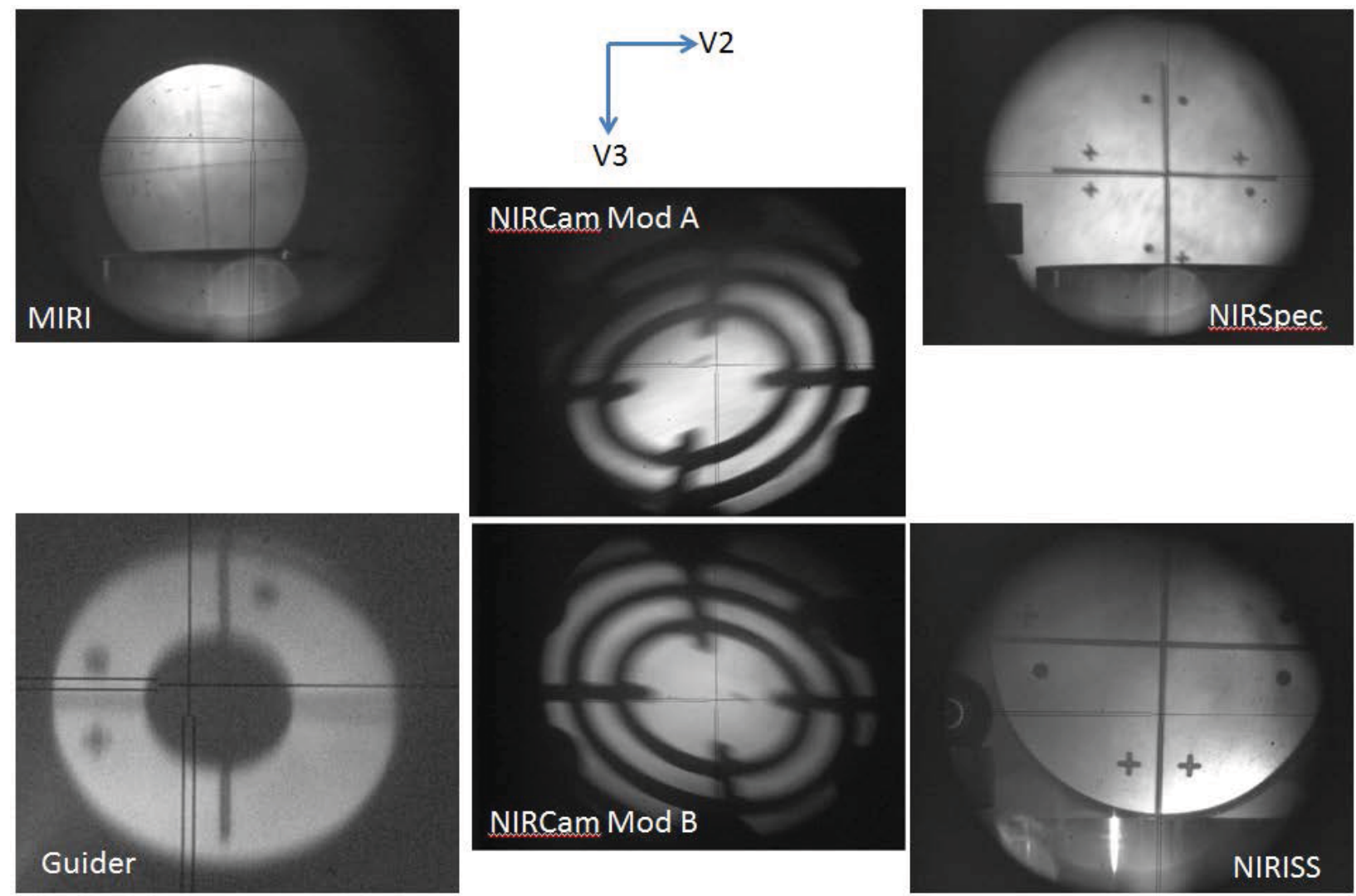

Figure 7 PAR images for all instrument PARs. The alignment telescope crosshair represents the nominal SI ambient pupil location, but offsets are expected from known calibrations and various wheel mechanism effects.

Uncertainties for all measurements are calculated via the student-t method. Typically, the uncertainties are based on five independent sets of measurements obtained at each instrument station.

The VCS orientation is shown in Figure 8 with respect to the ISIM structure. The frame shown in the figure is for approximate orientation reference and does not represent the actual origin. The directions of roll, pitch and yaw are used to report the cube vector results. 


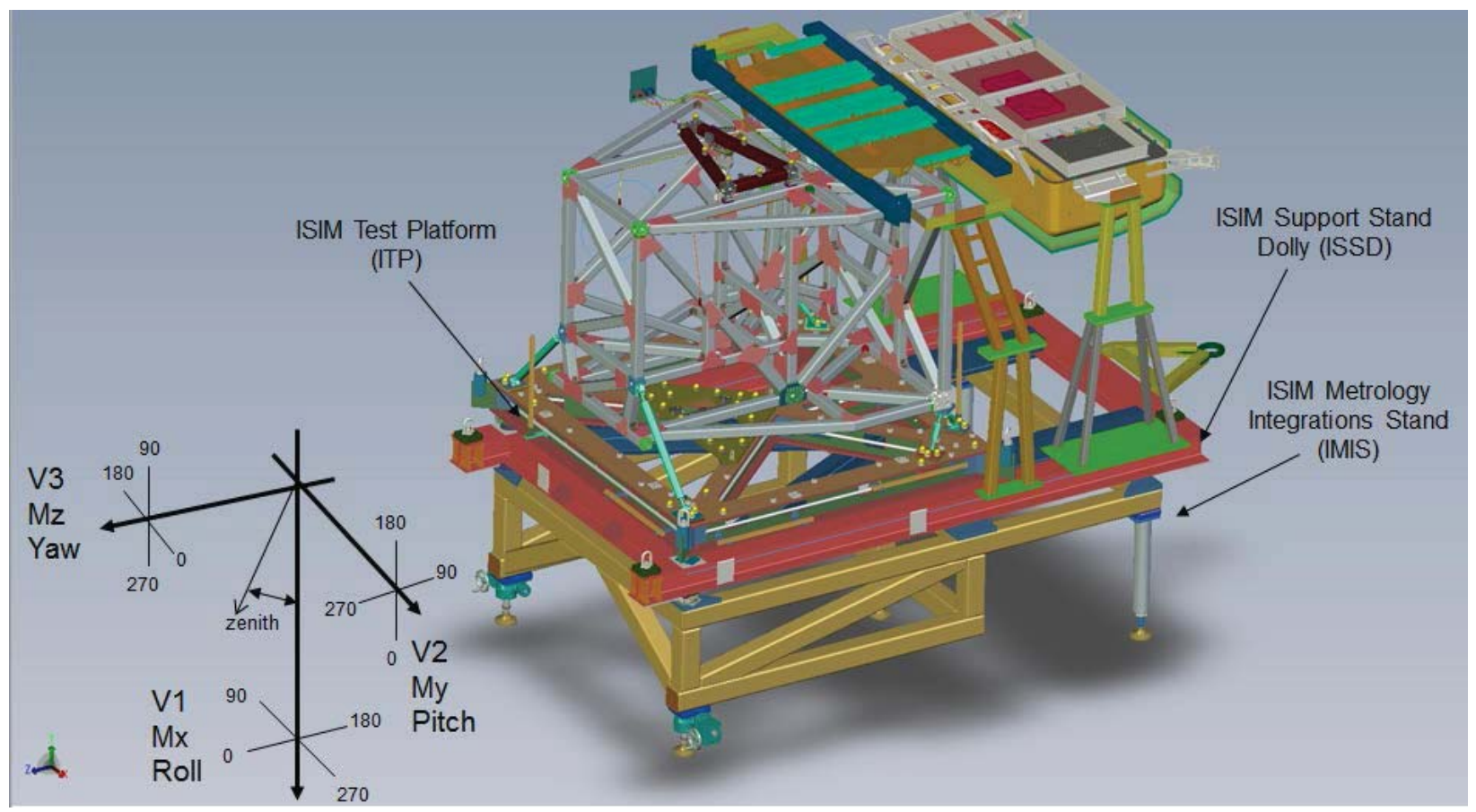

Figure 8 The VCS shown with respect to the unpopulated ISIM structure. The structure is mounted to the ITP and the test stand.

\section{RESULTS}

During the CV1 measurements only FGS and MIRI were integrated to the ISIM structure. The data presented here is from the pre-CV2 testing which has all of the SIs installed. The FEM differences of the SI OB values account for the change in loading conditions between the measurements on the ASMIF to the measurements on the ISIM structure. The results of the ISIM and SI TB/SMR values from pre-CV2 are summarized in Table 2. The "delta postCV1 FEM corrected" column summarizes differences of the ISIM target nest values between the partially loaded ISIM (i.e., just MIRI and FGS installed) with FEM predictions to the actual measured values of the fully loaded structure during pre-CV2 measurements. The "delta ASMIF FEM corrected" column is the difference between the ASMIF FEM corrected values to the as-measured values of the SIs on ISIM. The FEM predictions were applied to the measurements of the unloaded ISIM structure. Differences in the SIIP fabrication from the ISIM to ASMIF have also been accounted for analytically. The MIRI shield blocks line of sight to all MIRI nests and cube targets so the results shown in comparisons were made between MIRI measurements and pre-CV1 ASMIF measurements. Tables 2 and 3 show good agreement with the FEM predictions. Most values are below the pass/fail requirements (Figure 2 and 3). There are a few values that slightly exceed the requirements, but are not of concern because they are slightly over the pass/fail values. 
Table 2 Pre-CV2 ISIM and SI target deltas. MIRI deltas are in the VOTE-ISIM-PreCV1-A frame. Highlighted values are at or above the pass/fail requirements.

\begin{tabular}{|c|c|c|c|c|c|c|c|}
\hline \multirow{2}{*}{\multicolumn{2}{|c|}{$\frac{\text { Frame:VOTE-ISIM-PreCV2-A }}{\text { LR/LT measurments }}$}} & \multicolumn{3}{|c|}{ Delta Post CV1 FEM corrected } & \multicolumn{3}{|c|}{ Delta ASMIF FEM corrected } \\
\hline & & \multicolumn{2}{|c|}{$\mathrm{V} 1(\mathrm{~mm}) \quad \mathrm{V} 2(\mathrm{~mm})$} & V3 (mm) & \multicolumn{2}{|c|}{$\mathrm{V} 1(\mathrm{~mm}) \mathrm{V} 2(\mathrm{~mm})$} & V3 (mm) \\
\hline \multirow{7}{*}{ 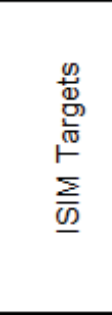 } & IST-B20 & 0.000 & -0.013 & 0.005 & & & \\
\hline & IST-B21 & 0.003 & 0.006 & -0.013 & & & \\
\hline & IST-B22 & -0.010 & -0.014 & -0.002 & & & \\
\hline & IST-B23 & -0.009 & -0.018 & 0.001 & & & \\
\hline & IST-B31 & -0.025 & 0.007 & 0.006 & & & \\
\hline & IST-B33 & 0.032 & 0.004 & -0.010 & & & \\
\hline & IST-B35 & -0.009 & -0.012 & -0.003 & & & \\
\hline \multirow{4}{*}{ 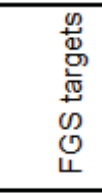 } & FGS-OB-F1 & 0.044 & -0.052 & -0.029 & 0.011 & 0.101 & 0.022 \\
\hline & FGS-OB-F2 & -0.007 & -0.029 & -0.028 & 0.026 & 0.064 & 0.032 \\
\hline & FGS-OB-F3 & -0.033 & -0.018 & -0.029 & 0.009 & 0.051 & 0.038 \\
\hline & FGS-OB-F5 & -0.031 & -0.023 & -0.020 & 0.014 & 0.005 & -0.007 \\
\hline \multirow{8}{*}{ 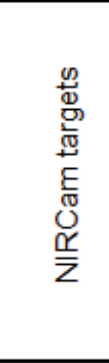 } & MODA +V1_1 & & & & -0.064 & -0.027 & 0.030 \\
\hline & MODA +V1_3 & & & & -0.053 & -0.054 & 0.030 \\
\hline & MODA +V3_4 & & & & -0.072 & -0.017 & 0.065 \\
\hline & MODA -V1_3 & & & & -0.119 & 0.034 & 0.057 \\
\hline & Module $\mathrm{A}+\mathrm{V} 3$ hole & & & & -0.043 & 0.023 & 0.048 \\
\hline & Module A -V1 hole & & & & -0.103 & 0.000 & 0.054 \\
\hline & Module $\mathrm{B}+\mathrm{V} 3$ hole & & & & -0.040 & 0.002 & 0.043 \\
\hline & Module B -V1 hole & & & & -0.071 & 0.008 & 0.058 \\
\hline \multirow{6}{*}{ 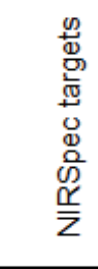 } & OBBP-LTT-01 & & & & -0.058 & -0.021 & -0.030 \\
\hline & OBBP-LTT-02 & & & & -0.027 & -0.002 & -0.047 \\
\hline & OBBP-LTT-03 & & & & -0.010 & -0.015 & -0.054 \\
\hline & OBBP-LTT-04 & & & & -0.044 & -0.020 & -0.056 \\
\hline & OBBP-LTT-05 & & & & -0.023 & 0.005 & -0.059 \\
\hline & OBBP-LTT-06 & & & & -0.032 & -0.110 & -0.035 \\
\hline \multirow{4}{*}{ 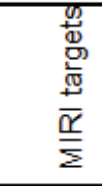 } & MIRI-OB-FA & & & & 0.016 & 0.045 & -0.077 \\
\hline & MIRI-OB-FB & & & & 0.035 & -0.002 & -0.040 \\
\hline & MIRI-OB-FC & & & & 0.033 & 0.000 & -0.033 \\
\hline & MIRI-OB-FD & & & & 0.062 & 0.026 & -0.042 \\
\hline
\end{tabular}


Table 3 Cube face direction results for the ISIM primary cube as well as the ITP and SI cubes. Greyed out blocks are rotations about the direction of the cube face (i.e., meaningless). Since the cube face is close to that particular axis the value cannot be calculated accurately. Highlighted values are at or above the pass/fail requirements but are not of concern because they are barely above the requirements.

\begin{tabular}{|c|c|c|c|c|c|c|c|c|c|}
\hline \multirow{2}{*}{$\begin{array}{l}\text { Theodolite FEM Deltas } \\
\text { Cube Vector }\end{array}$} & \multicolumn{3}{|c|}{ Roll about V1 } & \multicolumn{3}{|c|}{ Pitch about V2 } & \multicolumn{3}{|c|}{ Yaw about V3 } \\
\hline & deg & $\min$ & $\mathrm{sec}$ & deg & $\min$ & $\mathrm{sec}$ & deg & $\min$ & $\mathrm{sec}$ \\
\hline ISIM B21 -V2 & 0 & 0 & -2 & & & & 0 & 0 & 2 \\
\hline ISIM B33 -V3 & 0 & 0 & 1 & 0 & 0 & -5 & & & \\
\hline ITP OC4 +V2 & 0 & 0 & 1 & & & & 0 & 0 & -5 \\
\hline ITP OC4 +V3 & 0 & 0 & 2 & 0 & 0 & -10 & & & \\
\hline FGS-OC-F1 +V2 & 0 & 0 & 10 & & & & 0 & 0 & -27 \\
\hline FGS-OC-F1 -V3 & 0 & 0 & -4 & 0 & 0 & 18 & & & \\
\hline FGS-OC-F2 +V2 & 0 & 0 & -2 & & & & 0 & 0 & -32 \\
\hline FGS-OC-F2 -V3 & 0 & 0 & -2 & 0 & 0 & 13 & & & \\
\hline NIRCam-OC-B +V3 & 0 & 0 & 0 & 0 & 0 & -5 & & & \\
\hline NIRCam-OC-B -V1 & & & & 0 & 0 & -3 & 0 & 0 & 40 \\
\hline NS-OC-01+V3 & 0 & 0 & 33 & 0 & 0 & -9 & & & \\
\hline NS-OC-01 +V1 & & & & 0 & 0 & -9 & 0 & 0 & -1 \\
\hline NS-OC-02 -V3 & 0 & 0 & -39 & 0 & 0 & 4 & & & \\
\hline NS-OC-02 -V1 & & & & 0 & 0 & 3 & 0 & 0 & 19 \\
\hline MIRI-OC-F1 -V2 & 0 & 0 & -9 & & & & 0 & 0 & 22 \\
\hline MIRI-OC-F1 -V3 & 0 & 0 & -21 & 0 & 0 & 13 & & & \\
\hline MIRI-OC-F2 -V2 & 0 & 0 & 24 & & & & 0 & 0 & 44 \\
\hline MIRI-OC-F2 -V3 & 0 & 0 & 0 & 0 & 0 & 13 & & & \\
\hline
\end{tabular}

The PAR results are summarized below in Figures 9 and 10. The pass/fail requirement is $0.239 \mathrm{~mm}$ (2-sigma) and is shown as error bars on the charts. Data are shown from all tests to date. "Post- $S$ " is the post-shipment testing with the SI mounted to the ASMIF. The results shown in the charts are based on the difference between each measurement set and the "pre-CV1 prime" test. The values are in units of the SI's entrance pupil space (i.e., telescope exit pupil space). Data is limited for NIRSpec, because it was not integrated to ISIM until recently. NIRCam results were not available at the time of this publication. There are offsets in both V2 and V3 between the sets of measurements for all SIs, including between the ASMIF and integrated ISIM measurements. For the ASMIF measurements, the SIs are orientated with V1 horizontal, while, for the ISIM measurements, $+\mathrm{V} 1$ is down. The measurements have been corrected for expected gravity-induced movements of the Sls. The changes in V2 and V3 are mostly within the 2-sigma measurement uncertainties. Cases where there appears to be a larger-thanexpected offset are generally attributable to unknown systematic errors, like residual errors in the calculated gravity offsets and small internal offsets of the SI PAR. In either case, the measured offsets are compatible with the overall budget for pupil shears between the JWST telescope pupil and internal SI cold stops. The effect of differences in SIIP alignment have been removed by best-fitting and transforming the SI nest targets (from ASMIF) to the SI nest targets on ISIM. FGS Guider, MIRI, and NIRSpec show no significant pupil alignment change throughout all measurement sets. However, NIRISS does show a measureable change, primarily in V2, between "pre-CV1" and "post-CV1." The root cause is being investigated. The NIRISS PAR location will be measured postCV2 for further trending. 


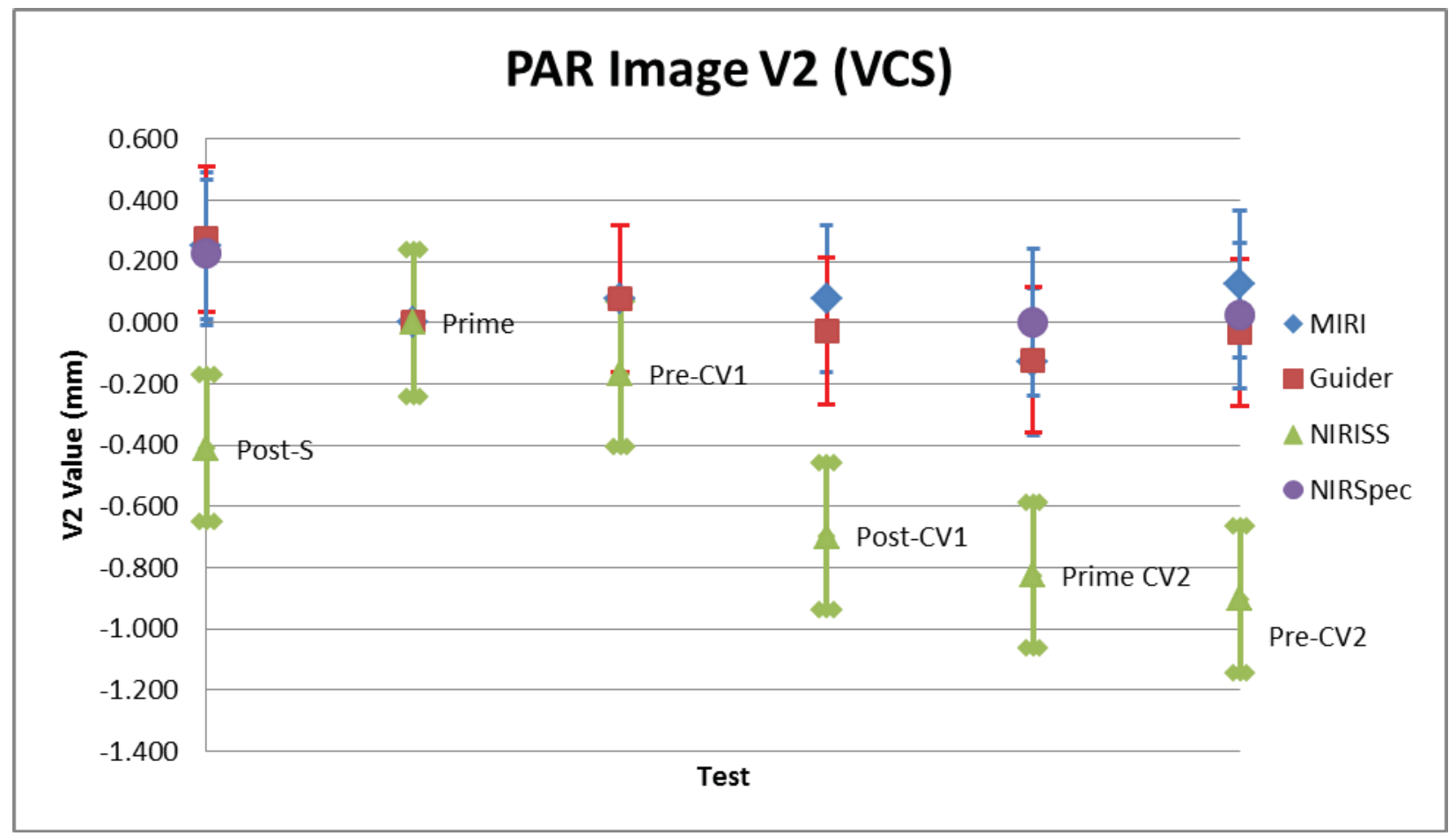

Figure 9 PAR image locations along V2. All tests are a delta from the Pre-CV1 Prime (labeled "prime") measurements.

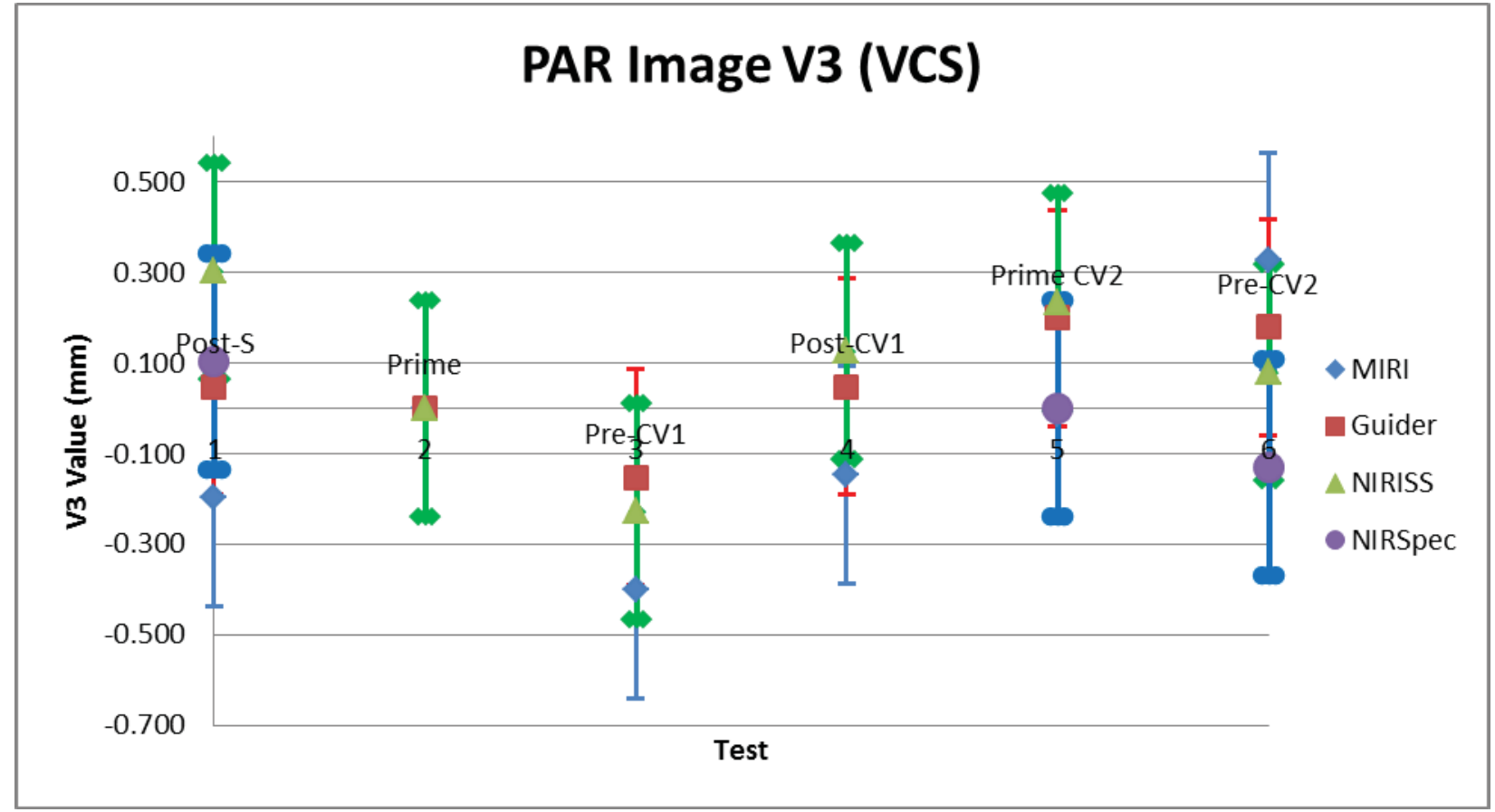

Figure 10 PAR image locations along V3. All tests are a delta from the Pre-CV1 Prime (labeled “prime") measurements. 


\section{CONCLUSION}

We have successfully verified that the SI-level alignment target calibration is in good agreement with measured SI OB locations on ISIM element to better than the required pass/fail values. The measurement process described in this paper will be repeated during ISIM-level I\&T to trend any potential alignment change due to thermal cycling, vibration and acoustic exposure, and then after SI work during I\&T. This work over the next year and a half will result in a concise database of trended values for the SIs and the structure throughout various integrated ISIM environmental test evolutions, which will inform the JWST as-built error analysis.

\section{REFERENCES}

[1] www.jwst.nasa.gov

[2] R. Lundquist et al., "Status of the James Webb Space Telescope integrated science instrument module" Proc. SPIE 8442, 2012.

[3] http://www.stsci.edu/jwst/instruments/nirspec

[4] http://www.stsci.edu/jwst/instruments/fgs

[5] http://www.stsci.edu/jwst/instruments/nircam

[6] http://www.stsci.edu/jwst/instruments/miri

[7] B. Bos et al., "Pupil alignment reference (PAR) for the Mid-Infrared Instrument (MIRI) for optical alignment and verification on the Integrated Science Instrument Module (ISIM) in James Webb Space Telescope (JWST)" Proc. SPIE 7433, 2009.

[8] J. Johnston, et al.,"Cryogenic thermal distortion performance characterization for the JWST ISIM structure" Proc. SPIE 8150, 2011.

[9] J. Johnston, E. Cofie.,"Cryogenic thermal distortion model validation for the JWST ISIM structure" Proc. SPIE 8150, 2011.

[9] JWST-RPT-008175 ISIM Error Budget Report

[11] J. Burge et al., "Use of a commercial laser tracker for optical alignment," Proc. SPIE 6676, 2007.

[12] Leica Geosystems, Inc., Norcross, Ga.

[13] M.Menon et al., "Remote Metrology, Mapping, and Motion Sensing of Plasma Facing Components Using FM Coherent Laser Radar," Fusion Engineering Design 58-59, 495-498, 2001.

[14] Nikon Metrology NV, Leuven, Belgium

[15] New River Kinematics, Inc., Williamsburg, Va.

[16] S. Sandwith \& R. Predmore, "Real-time 5-micron Uncertainty with Laser Tracking Interferometer Systems using Weighted Trilateration," New River Kinematics, Inc. Williamsburg, Va.

[17] Spatial Analyzer User Manual, page 322, v. 2013.12.09.

[18] W. Eichhorn, “Optical alignment measurements at Goddard Space Flight Center," Applied Optics 21, $3891,1982$.

[19] J. Hayden et al., "Measurements and Analysis used for the Determination of the James Webb Space Telescope Integrated Science Instrument Module Vehicle Coordinate System," Coordinate Metrology Society Conference, July 2010.

[20] http://imagej.nih.gov/ij/ 